\title{
Thermal Lattice Boltzmann Model for Natural Convection in an Inclined Cavity Packed with Porous Material
}

\author{
Atia Abdelmalek $^{1}$ and Mohammedi Kamal ${ }^{2}$ \\ 1. Mechanical Engineering Departement, Faculty of Sciences and Technology, University of Hamma Lakhdar, El-oued 39000, Algeria \\ 2. Laboratory of Energetic, Mechanics and Engineering, Faculty of Sciences of Engineer, University of M'hamed Bougara, \\ Boumerdes 35000, Algeria
}

\begin{abstract}
In the present work, the LBM (Lattice-Boltzmann method) is used to simulate natural convection in an inclined open ended square cavity filled with porous material. The cavity is submitted to heating and cooling from two opposite sides with constant temperatures. The double-population approach is used to simulate hydrodynamic and thermal fields. The effect of a porous medium is taken into account by introducing the porosity into the equilibrium distribution function and adding a force term to the evolution equation. The Brinkman-Forchheimer equation, which includes the viscous and inertial terms, is applied to predict the heat transfer and fluid dynamics in the non-Darcy regime. The present model is validated with the previous literature. A comprehensive parametric study of natural convective flows is performed for various values of inclined angle.
\end{abstract}

Key words: Lattice boltzmann method, natural convection, porous cavity, Brinkman-Forchheimer equation.

\section{Introduction}

There is no need to say that LBM (Lattice Boltzmann Method) are in high pace development and have become a powerful method for simulation fluid flow and transport problems for single and multiphase flows [1,2]. In this work, the method is applied for natural convection in open ended porous cavities.

Natural convection has lots of applications in both nature and engineering, such as the cooling of electronic devices and heat transfer improvement in heat exchanger apparatuses and petroleum reservoirs. Because cavities and slots are benchmark test cases, many researches using LBM [3, 4]. Natural convection in a square cavity and its fluid flow is a classical problem in mechanical engineering. Mohamad et al. [5] presented a detailed analysis of natural convection problem to use LBM; they demonstrated the abilities of the LBM in simulating natural convection. Open cavities are 2-D cavity that has an open side. These kinds of cavities have special

Corresponding author: Atia Abdelmalek, D.Sc. candidate, research fields: modelling and simulation in mechanical engineering. E-mail: abdelmalek-atia@univ-eloued.dz. physics in open side because of outgoing of flow from this side. Some research papers have been published on studies of buoyant flows and their heat transfer in open cavities [6]. Mohamad et al. [7] presented natural convection in an open ended cavity and slots, they analyzed the effect of aspect ratio of cavity on heat transfer rate. They presented a good procedure for simulating open boundaries in LBM.

Research on natural convection in enclosure packed with a porous medium is motivated by its wide applications in engineering, such as drying processes, chemical catalytic reactors and solar power collectors. Several models have been introduced for natural convection heat transfer in porous media. An excellent and comprehensive review has been given by Nield and Bejan [8]. The buoyancy-driven convection in a porous cavity is analyzed by D. Gao et al. [9]. A. Haghshenas et al. [4] studied numerically the steady natural convection in a square cavity filled with a porous medium via LBM.

\section{Thermal Lattice Boltzmann Method}

The thermal Lattice Boltzmann model utilizes two 
distribution functions, $f$ and $g$, for the flow and temperature fields, respectively. In this approach the fluid domain is discredited in uniform Cartesian cells. Each cell holds a fixed number of distribution functions. For this work the D2Q9 model has been used. This model is shown in Fig. 1.

For the D2Q9 lattice, the nine velocities $\vec{c}_{i}$ and their corresponding weights $w_{i}$ are calculated from Ref. [10]:

$$
\vec{c}_{i}=c\left\{\begin{array}{c}
0 \text { for } i=0 \\
\left(\cos \left[(i-1) \frac{\pi}{2}\right], \sin \left[(i-1) \frac{\pi}{2}\right]\right) \text { for } i=1,2,3,4 \\
\sqrt{2}\left(\cos \left[(i-5) \frac{\pi}{2}+\frac{\pi}{4}\right], \sin \left[(i-5) \frac{\pi}{2}+\frac{\pi}{4}\right]\right) \text { for } i=5,6,7,8
\end{array}\right.
$$

And the weighting factors are given by:

$$
\begin{gathered}
w_{0}=\frac{4}{9}, w_{1}=w_{2}=w_{3}=w_{4}=\frac{1}{9}, \\
\text { and } w_{5}=w_{6}=w_{7}=w_{8}=\frac{1}{36}
\end{gathered}
$$

It is to be noted that in the above equations, $c=$ $\frac{\Delta x}{\Delta t}$, and the weights satisfy the relation $\sum_{i=1}^{9} w_{i}=1$.

The Lattice Boltzmann equation with an external force $F$ can be written respectively for the fluid flow and temperature distribution as follows:

The $f(r, t)$ and $g(r, t)$ are calculated by solving the Lattice Boltzmann equation. By using BGK model, the general form of Lattice Boltzmann equation with an added force term can be written as:

For the flow field:

$$
\begin{gathered}
f_{i}\left(r+c_{i} \Delta t, t+\Delta t\right)= \\
f_{i}(r, t)\left[1-\omega_{v}\right]+\omega_{v} f_{i}^{e q}(r, t)+\Delta t . \mathrm{F}_{i}
\end{gathered}
$$

For the temperature field:

$$
\begin{gathered}
g_{i}\left(r+c_{i} \Delta t, t+\Delta t\right)= \\
g_{i}(r, t)\left[1-\omega_{T}\right]+\omega_{T} g_{i}^{e q}(r, t)
\end{gathered}
$$

where, $\Delta t$ denotes lattice time step, $c_{i}$ is the discrete lattice velocity in direction $i, \mathrm{~F}_{i}$ is the total body force in direction of lattice velocity, $\omega_{v}=\frac{\Delta t}{\tau_{v}}$ and $\omega_{T}=\frac{\Delta t}{\tau_{T}}$ denotes the non-dimensional relaxation time for the flow and temperature fields. The kinetic viscosity $v$ and the thermal diffusivity $\alpha$ are defined in terms of their respective relaxation times, i.e.

$$
v=c_{S}^{2}\left(\tau_{v}-1 / 2\right), \quad \text { and } \quad \alpha=c_{S}^{2}\left(\tau_{T}-1 / 2\right),
$$
respectively. The local equilibrium distribution for flow and temperature fields is as follows respectively.

$$
f_{i}^{e q}=w_{\mathrm{i}} \rho\left[1+\frac{c_{i} \cdot u}{c_{s}^{2}}+\frac{1}{2} \frac{\left(c_{i} \cdot u\right)^{2}}{\epsilon c_{s}^{4}}-\frac{1}{2} \frac{u^{2}}{\epsilon c_{s}^{2}}\right]
$$

where, $\epsilon$ is the porosity of the material.

$$
g_{i}^{e q}=w_{\mathrm{i}} T\left[1+\frac{c_{i} \cdot u}{c_{s}^{2}}+\frac{1}{2} \frac{\left(c_{i} \cdot u\right)^{2}}{c_{s}^{4}}-\frac{1}{2} \frac{u^{2}}{c_{s}^{2}}\right]
$$

In LBM Eqs. (3) and (4) are solved in two important steps that are called collision and streaming step. Collision step is as follows for flow field and temperature field respectively [10]:

$$
\begin{gathered}
f_{i}(r, t+\Delta t)=f_{i}(r, t)\left[1-\omega_{v}\right]+ \\
\omega_{v} f_{i}^{e q}(r, t)+\Delta t . \mathrm{F}_{i} \\
g_{i}(r, t+\Delta t)=g_{i}(r, t)\left[1-\omega_{T}\right]+\omega_{T} g_{i}^{e q}(r, t)(8)
\end{gathered}
$$

Streaming step can be written as follows:

$$
\begin{aligned}
& f_{i}\left(r+c_{i} \Delta t, t+\Delta t\right)=f_{i}(r, t+\Delta t) \\
& g_{i}\left(r+c_{i} \Delta t, t+\Delta t\right)=g_{i}(r, t+\Delta t)
\end{aligned}
$$

Macroscopic variable can be calculated in terms of these variables, with the following formula:

Flow density:

Momentum:

$$
\rho=\sum_{i=0}^{9} f_{i}
$$

$$
\rho u=\sum_{i=0}^{9} f_{i} c_{i}
$$

Temperature:

$$
T=\sum_{i=0}^{9} g_{i}
$$

The total body is added as an extra source term to Eq. (14),

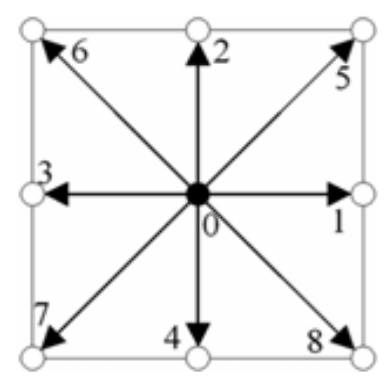

Fig. 1 Lattice arrangements for 2-D problems, D2Q9. 


$$
\mathrm{F}_{i}=w_{\mathrm{i}} \rho\left(1-\frac{w_{v}}{2}\right)\left[\frac{3 c_{i} \cdot F}{c^{2}}+\frac{9 u F: c_{i} c_{i}}{\epsilon c^{4}}-\frac{3 u F}{\epsilon \cdot c^{2}}\right]
$$

where, $\mathrm{F}=\mathrm{F}_{\text {buoyancy force }}+\mathrm{F}_{\text {porous material }}$.

With the Boussinesq approximation the buoyancy force modeled as:

$$
\begin{gathered}
\mathrm{F}_{\text {buoyancy force }}= \\
\epsilon g \beta\left(T-T_{m}\right)\left(c y_{i} \cos \varphi+c x_{i} \sin \varphi\right)
\end{gathered}
$$

where, $T_{m}=\left(T_{h}+T_{c}\right) / 2$.

$$
\mathrm{F}_{\text {porous material }}=-\frac{\epsilon v}{K} u-\frac{\epsilon 1.75}{\sqrt{150 \varepsilon K}}|u| u
$$

where, $g, \beta, \epsilon, K$, and $T_{m}$ are gravitational acceleration, thermal expansion coefficient, porosity of the medium, permeability and reference temperature.

\section{Position of the Problem and Boundary Conditions}

The studied configuration is sketched in Fig. 2. It consists of a square cavity of length $\mathrm{H}$ with two partially heated walls, located on two opposite walls, and maintained at constant temperatures $T_{h}$ and $T_{c}$ $\left(T_{h}>T_{c}\right)$, respectively. The cavity is inclined at an angle $\varphi$ with respect to the horizontal and filled with porous material; the east side is considered as open ended.

The distribution functions out of the domain are known from the streaming process. The unknown distribution functions are those toward the domain. Fig. 3 shows the unknown distribution function, which needs to be determined, as dotted lines [7].

Flow:

Bounce back boundary condition is used on the solid boundaries (west, north and south boundaries). At the east open boundary, the following condition is used,

$$
f_{6 n}=f_{6 n-1}, f_{3 n}=f_{3 n-1}, f_{7 n}=f_{7 n-1}
$$
where, $n$ is the lattice on the boundary and $n-1$, is the lattice inside the cavity adjacent to the boundary.

Temperature:

Bounce back boundary condition (adiabatic) is used on the north and south of the boundaries. Temperature at the west wall is known, $\mathrm{Tw}=1.0$. Since we are using D2Q9, the unknown are $g 1, g 5$ and $g 8$, which are evaluated as,

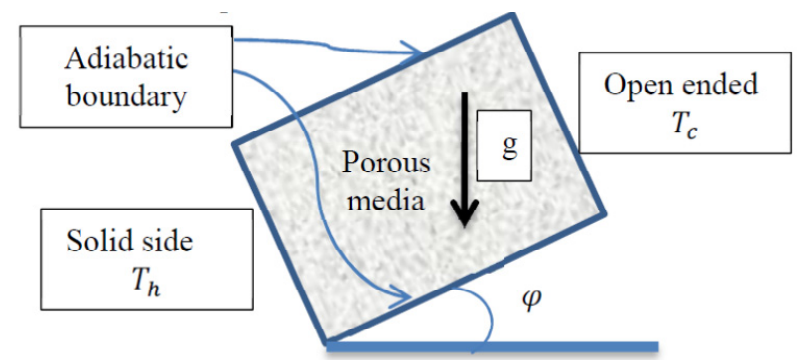

Fig. 2 Studied configuration.

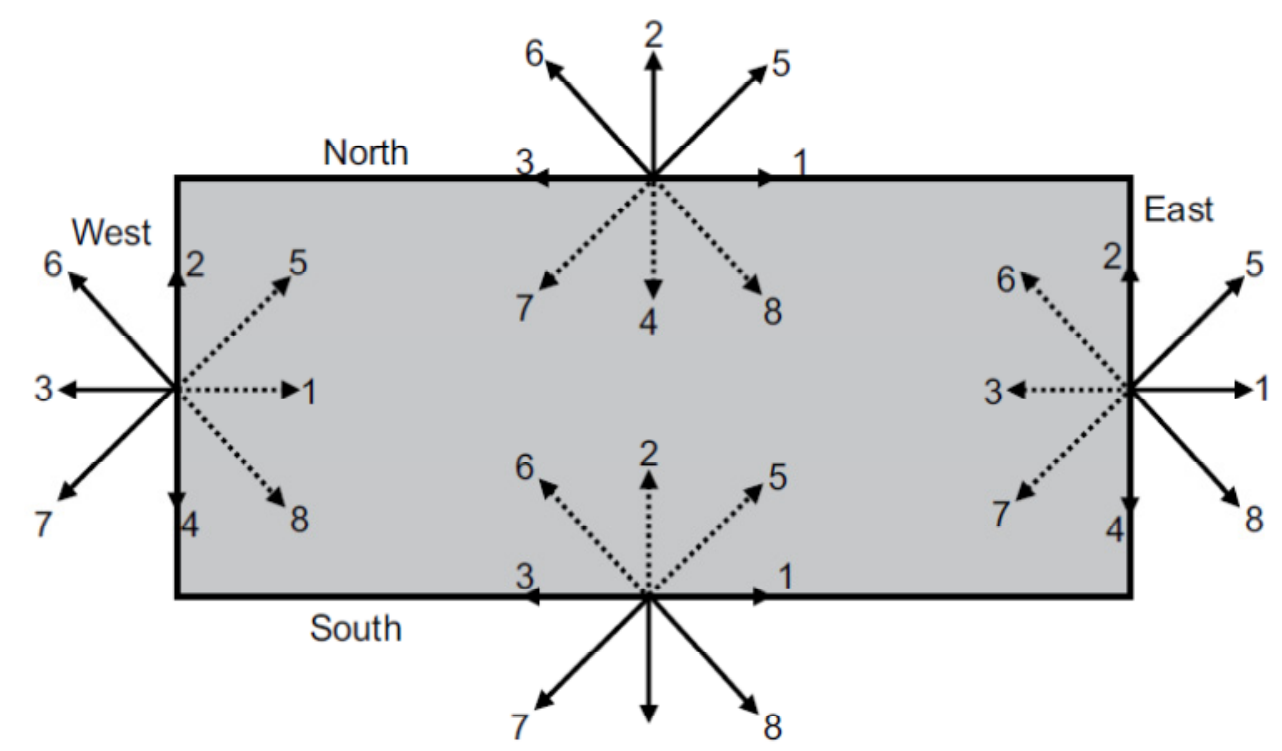

Fig. 3 Domain boundaries and direction of streaming velocities. 


$$
\begin{aligned}
& g 1=T w *(w 1+w 3)-g 3 \\
& g 5=T w *(w 5+w 7)-g 7 \\
& g 8=T w *(w 8+w 6)-g 6
\end{aligned}
$$

The boundary condition for east wall needs special treatment, since prior to solution, the advected velocity direction is not known. It is assumed that if the flow is penetrating into the cavity $(u<0)$, then the temperature should be ambient, $\mathrm{T}=0$, and if the flow leaving the cavity $(u>0)$, it is assumed that there is no heat diffusion, i.e., gradient of temperature is negligible.

For east boundary, the distributions function, $g 6, g 3$ and $g 7$, are evaluated as,

$$
\begin{aligned}
& \text { if } u<0 \text { then }: \\
& g 6, n=0-g 8, n \\
& g 3, n=0-g 1, n \\
& g 7, n=0-g 5, n \\
& \text { if } u>0 \text { then }: \\
& g 6, n=g 6, n-1 \\
& g 3, n=g 3, n-1 \\
& g 7, n=g 7, n-1
\end{aligned}
$$

\section{Results and Discussion}

In this work the problem of natural convection heat transfer in an inclined open ended cavity packed with porous material is simulated. The cavity has two horizontal insulated walls, a west wall with constant temperature and an open east side. The temperature of west wall is equal to unity that is more than the ambient zero temperature. One point must be noticed that this temperature is dimensionless in Lattice scale. In the present study to simulate natural convection in inclined cavity, the gravity acceleration ( $g$ ) is rotated counter clockwise. The negative values of rotating angel mean rotation is clockwise. To validate the numerical method, two different cases are tested:

(1) Natural convection in an open-ended cavity without porous medium, and without rotating angel.

(2) Natural convection in an open-ended cavity with porous medium, and without rotating angel.
The results are compared with the findings of Mohamad et al. [7] for case (1) and of A. Haghshenas et al. [4] for case (2).

Tables 1 and 2 summarize the average Nusselt numbers obtained by the present work and compared with the previous literature.

As it can be seen from these tables, in each case, the results are considerably consistent with those in the literature and these comparisons.

Hence, it is concluded that LBM with the suggested boundary condition at the opening of the cavity can produce reliable results.

Figs. 4 and 5 show streamlines and isotherms, respectively, comparison between the present study and that presented by Mohamad et al. [7] for Ra $=10^{4}$. The flow enters from the lower half portion of the cavity and leaves from the upper half of the opening.

Now, the problem of natural convection heat transfer in an inclined open porous cavity was solved for different angles, when Rayligh number is fixed to $10^{4}$, Prantdl number ( $\left.P r\right)$ is fixed to 0.71 , porosity is fixed to 0.4, and Darcy number is fixed to 0.01 . Streamline and Temperature contours are plotted and average Nusselt number on closed west wall is investigated.

Figs. 6 and 7 Streamline and Temperature contours are plotted for different rotation angle, the effect of rotation on Streamline and Temperature contours can be seen obviously.

Table 1 Average Nusselt number comparison for differents Rayligh number and $\mathrm{Pr}=0.71$ (case 1).

\begin{tabular}{lll}
\hline $\mathrm{Ra}$ & Mohamad et al. (FV) & LBM (present) \\
\hline $10^{4}$ & 3.264 & 3.249202 \\
$10^{5}$ & 7.261 & 7.061324 \\
$10^{6}$ & 14.076 & 14.045800 \\
\hline
\end{tabular}

Table 2 Average Nusselt number comparison for differents Rayligh number and $\operatorname{Pr}=1.0, \mathrm{Da}=0.01$ and $\varepsilon=0.4$ (case 2).

\begin{tabular}{lll}
\hline $\mathrm{Ra}$ & A. Haghshenas et al. (LBM) & LBM (present) \\
\hline $10^{4}$ & 1.541 & 1.678586 \\
$10^{5}$ & 4.294 & 4.327931 \\
$10^{6}$ & 9.432 & 9.598819 \\
\hline
\end{tabular}




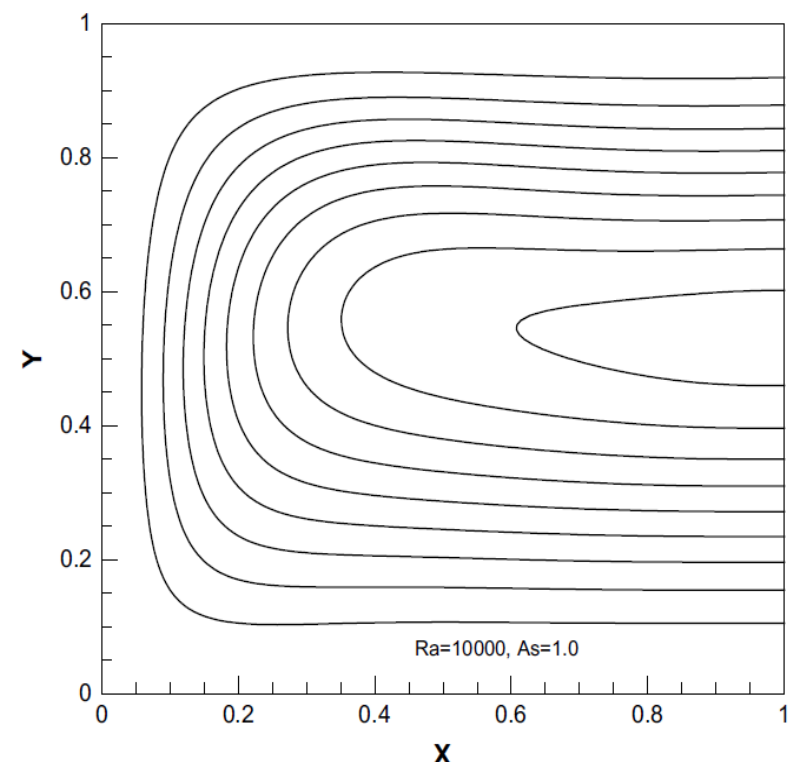

(a) Mohamad et al. [7]

Fig. 4 Streamlines for $\mathrm{Ra}=10^{4}$ and aspect ratio of 1.0 .

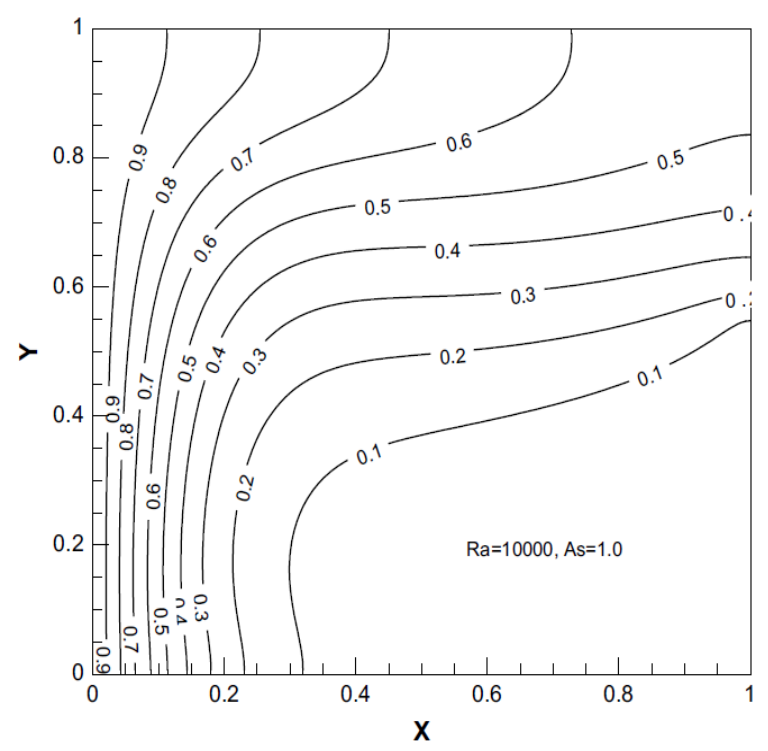

(a) Mohamad et al. [7]

Fig. 5 Isotherms for $\mathrm{Ra}=\mathbf{1 0 4}$ and aspect ratio of $\mathbf{1 . 0}$.

Table 3 shows that rotation angle has low effect on average Nusselt in the low Rayligh number $(\mathrm{Ra}=$ $\left.10^{4}\right)$.

\section{Conclusion}

In the present work, simulations of flow and temperature fields that arise due to natural convection in an open-ended inclined square cavity filled with a

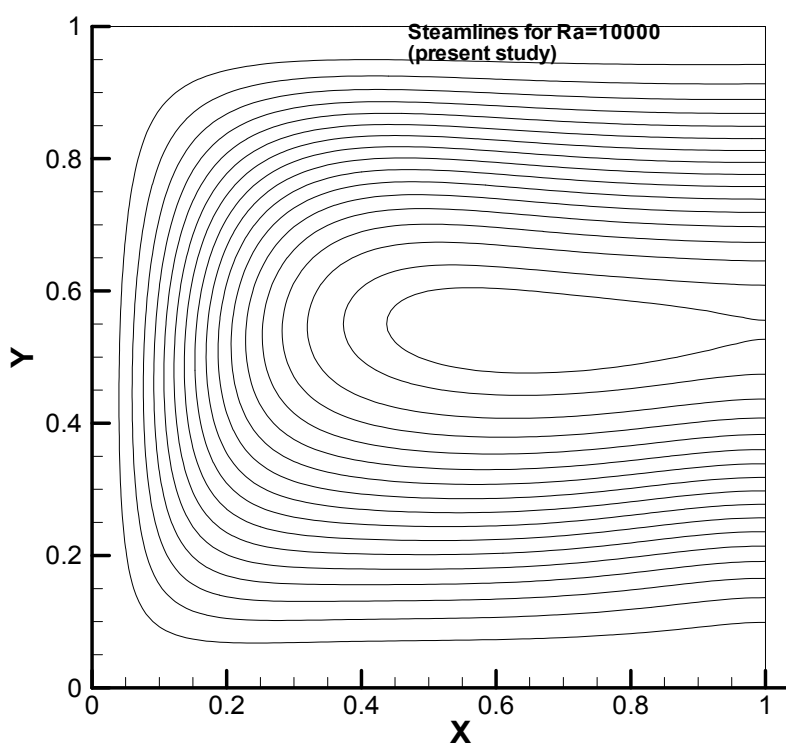

(b) Present study

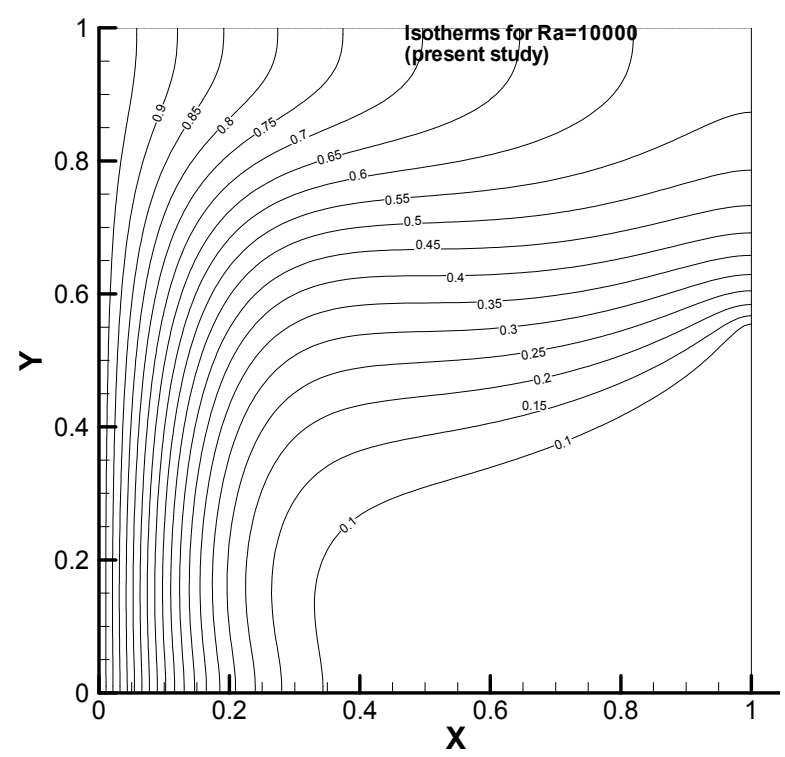

(b) Present study

porous medium have been carried out. The double-population approach, the LBM were employed. The influence of rotation angle was considered by adding a force term to the evolution equation. To predict the heat transfer and fluid dynamics in the non-Darcy regime, the Brinkman-Forchheimer equation was applied. The numerical method was validated by two different cases and a good agreement 

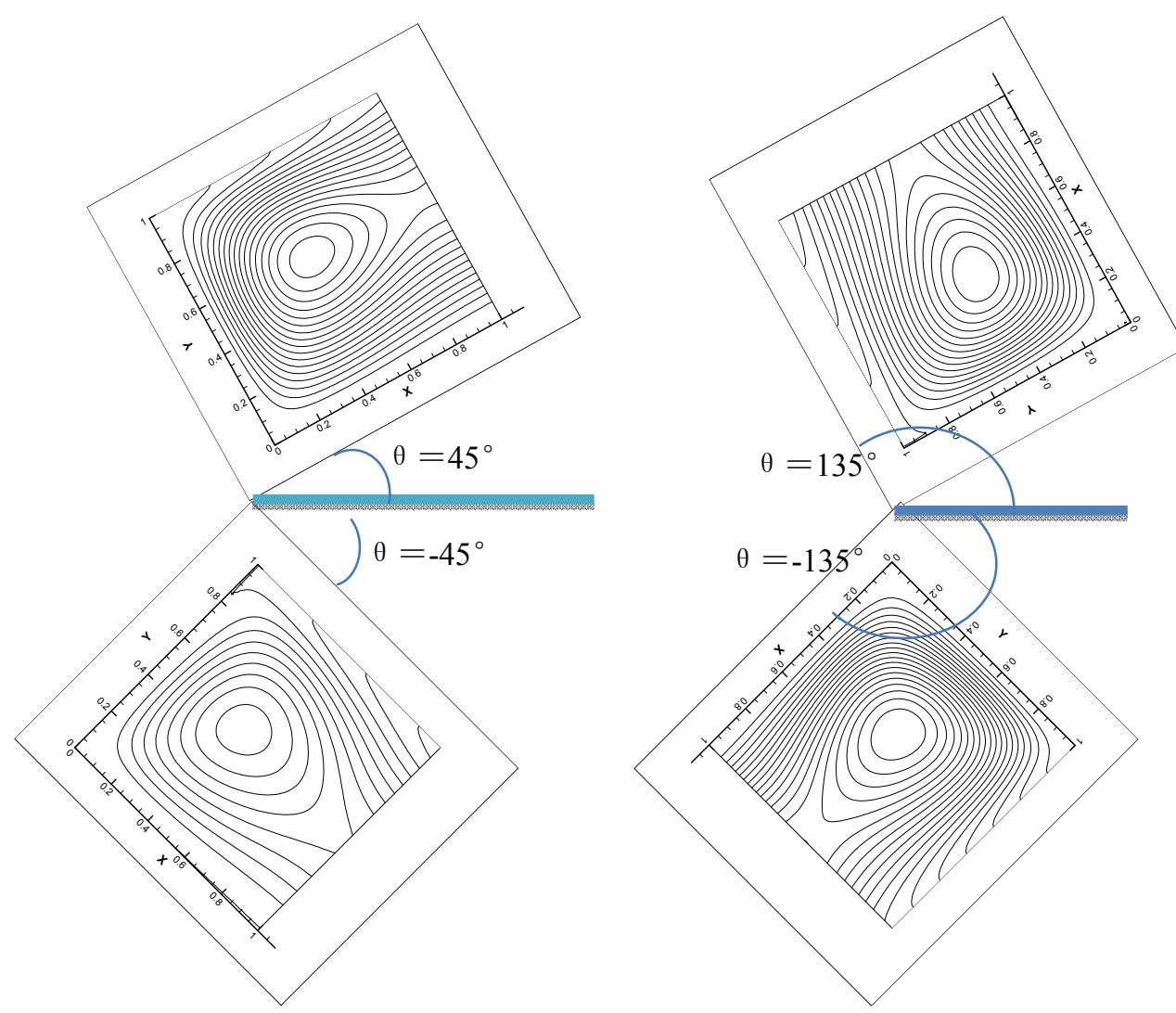

Fig. 6 Streamlines for different rotation angle.
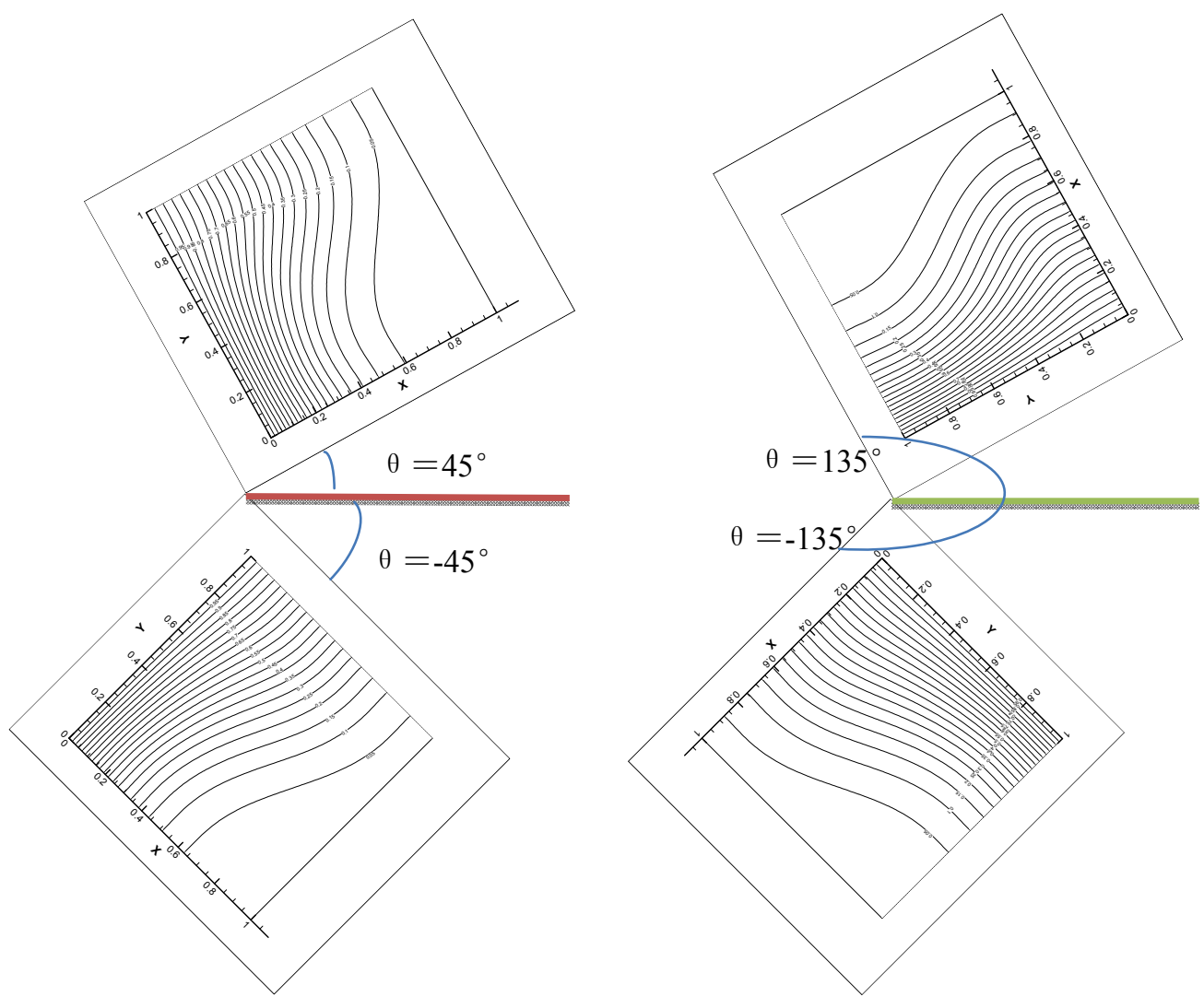

Fig. 7 Isotherms for different rotation angle. 
Table 3 Average Nusselt number comparison for different rotation angle.

\begin{tabular}{ll}
\hline$\theta$ & $\mathrm{Nu}$ \\
\hline $45^{\circ}$ & 2.312473 \\
$-45^{\circ}$ & 2.294909 \\
$135^{\circ}$ & 2.312482 \\
$-135^{\circ}$ & 2.294900 \\
\hline
\end{tabular}

was observed. It was found that, for low Rayleigh numbers, the average Nusselt number is almost independent of rotation angle.

\section{References}

[1] Mohamad, A. 2007. “Applied Lattice Boltzmann Method for Transport Phenomena, Momentum." The Canadian Journal of Chemical Engineering 85 (6): 946-7.

[2] Succi, S. 2001. The Lattice Boltzmann Equation: For Fluid Dynamics and Beyond: Oxford University Press.

[3] Fattahi, E., Farhadi, M., and Sedighi, K. 2010. "Lattice Boltzmann Simulation of Natural Convection Heat Transfer in Eccentric Annulus." International Journal of Thermal Sciences 49: 2353-62.

[4] Haghshenas, A., Nasr, M. R., and Rahimian, M. 2010.
"Numerical Simulation of Natural Convection in an Open-Ended Square Cavity Filled with Porous Medium by Lattice Boltzmann Method." International Communications in Heat and Mass Transfer 37: 1513-9.

[5] Mohamad, A., and Kuzmin, A. 2010. "A Critical Evaluation of Force Term in Lattice Boltzmann Method, Natural Convection Problem." International Journal of Heat and Mass Transfer 53: 990-6.

[6] Polat, O., and Bilgen, E. 2002. "Laminar Natural Convection in Inclined Open Shallow Cavities." International Journal of Thermal Sciences 41: 360-8.

[7] Mohamad, A., El-Ganaoui, M., and Bennacer, R. 2009. "Lattice Boltzmann Simulation of Natural Convection in an Open Ended Cavity." International Journal of Thermal Sciences 48: 1870-5.

[8] Nield, D. A., and Bejan, A. 2006. Convection in Porous Media: Springer.

[9] Gao, D., and Chen, Z. 2011. "Lattice Boltzmann Simulation of Natural Convection Dominated Melting in a Rectangular Cavity Filled with Porous Media." International Journal of Thermal Sciences 50: 493-501.

[10] Mohamad, A. A. 2011. Lattice Boltzmann Method: Fundamentals and Engineering Applications with Computer Codes: Springer. 